\title{
Postoperative Ileus in Elective Colorectal Surgery: Management Strategies
}

\author{
J. Ahmed, S. Mehmood and J. MacFie \\ Scarborough General Hospital, Scarborough \\ United Kingdom
}

\section{Introduction}

Postoperative ileus (POI) is a condition characterised by transient interruption of gut function following surgical intervention. Ileus develops due to impaired motility of the gastrointestinal tract in the absence of any mechanical bowel obstruction (Holte and Kehlet 2000). This results in disturbance of coordinated propulsive action leading to accumulation of luminal gas and fluid mainly within small bowel. Clinically it is manifested by abdominal distension, nausea, vomiting and diet intolerance (Lubawski and Saclarides 2008; Parvizi, et al. 2008).

Despite a number of advances in perioperative care and surgical techniques, POI remains one of the commonest challenges in surgery. Although most commonly seen following abdominal surgery, it is also associated with extra-abdominal operations such as cardiothoracic and orthopaedic procedures (Parvizi, et al. 2008; Stewart and Waxman 2010). Historically, POI has been regarded as an inevitable event after major abdominal surgery and it is considered the single largest factor prolonging the length of hospital stay (LOS) following gastrointestinal surgery (Lubawski and Saclarides 2008). It impacts greatly on patient's recovery even after uncomplicated abdominal surgery. Similarly, postoperative ileus is one of the commonest factors affecting healthcare costs in surgical patients. Furthermore, POI causes significant frustration amongst the patients and the surgeons alike (Asgeirsson, et al. 2010).

In recent years, some progress has been made in understanding of underlying causative mechanisms of POI. The multifaceted pathophysiology of POI includes neurogenic, hormonal, inflammatory and pharmacological factors which have been targeted to reduce the incidence and duration of POI. However, there is no definitive treatment to prevent this condition. Key principles in the prevention and management of POI pertain to minimising surgical trauma and preserving normal physiological functions, including gut function. Evidence suggests that this is best achieved by a multimodal approach involving a host of interventions in the perioperative period. Perhaps the most significant development in recent times is the formulation of enhanced recovery after surgery (ERAS) protocols. ERAS protocols provide a multimodal approach to optimising perioperative care and enhancing postoperative recovery. Likewise, minimally invasive surgery i.e. laparoscopic approach has been shown to provide benefits like reduction in length of stay in hospital. Similarly, newer pharmacological therapies (e.g. Mu-opioid receptor antagonist) have shown promising results, however, more scientific evidence is awaited in this area. 
In this chapter, we give an account of pathophysiology, natural history, and classification of ileus. Different strategies to prevent and manage POI such as ERAS protocols, minimally invasive surgery and pharmacological approaches are discussed.

\section{History and definitions of POI}

Ileus is a Greek word which literally means "obstructed" however it is referred to as a functional impediment without mechanical obstruction. POI has been a recognised condition for more than two centuries. The exact definition of POI has been a topic for debate for over a century. It was suggested by Finney in 1906 to divide the phenomnon of ileus into pathophysiological, mechanical, septic or adynamic ileus (Finney 1906). One hundred years later in 2006, the 'Postoperative Ileus Management Council' (PIMC) was formed by experts in the field of surgery and anaesthesia. The PIMC collected all scientific evidence and developed a consensus on the definition of POI. According to this panel POI is a:

"transient cessation of coordinated bowel motility after surgical intervention, which prevents effective transit of intestinal contents or tolerance of oral intake".

The PIMC categorised POI into primary and secondary ileus. Primary POI develops in the absence of any precipitating factors (i.e. absence of any complications), while secondary POI develops in the presence of a complication such as wound infection, intra-abdominal collection, anastomotic leak, and sepsis etc. In addition, a further three types of POI were described based on the part of gastrointestinal tract (GIT) involved. Type-I POI involves all part of the GIT and thus called pan-intestinal ileus. Clinically it is manifested as nausea and vomiting along with absence of bowel movement or passage of flatus. Type-II POI affects only the upper GIT and manifests as nausea and vomiting. These patients are able to tolerate food but are unable to pass flatus. Type-III POI comprises of ileus of the lower GIT. The patients may be able to tolerate food but are unable to pass flatus or have a bowel motion (Delaney C 2006).

The duration of POI and the cardinal features of its resolution are important aspects which need careful consideration. The minimum time period following surgery before ileus develops is not well defined. Animal and human models have shown that return of gut function occurs first of all in small bowel ( 24 hours) followed by stomach (48 hours) and colon (48 to 72 hours) (Lubawski and Saclarides 2008). It has been suggested that the colon may take up to 120 hours to resume normal peristaltic activities postoperatively. (Miedema and Johnson 2003).

The return of gut function is historically assessed by either of the presence of bowel sounds, passage of flatus or stool, or tolerance to oral food. Passage of flatus or stool and/or presence of bowel sounds are considered as the benchmark criteria for the recovery of bowel function. However, concerns have been expressed about the validity and accuracy in recording these signs. For example, presence of bowel sounds does not necessarily indicate return of propulsive activities of the whole bowel as they may only represent small bowel activity without colonic peristalsis (Holte and Kehlet 2000). In addition, accuracy of recording these features is usually low. To record presence of bowel sounds requires frequent auscultation which is practically difficult. Similarly, passage of flatus is also not the ideal end point. Some patients are simply not comfortable to report it whereas others may not be able to recall passing flatus in the postoperative peroid. The passage of stool is also 
considered to have low specificity. A patient might report having a bowel movement which would be representing distal bowel evacuation as opposed to global gastrointestinal tract function. Hence determination of the end of postoperative ileus is another contentious issue.

The combination of all three signs (i.e. presence of bowel sounds, passage of stool or flatus along with tolerance of oral feed) provides higher accuracy in confirming resolution of POI. Thus, based on the available evidence and PIMC consensus report the resolution of POI can be defined:

"the duration of POI is the time from the surgery until passage of stool or flatus and until oral feed that is tolerated and maintained during 24 hours".

However, it is important to appreciate that uncertainties in criteria for the resolution of POI have impact on the conclusions of various studies published in the literature (Boeckxstaens and de Jonge 2009).

\section{Pathophysiology of post-operative ileus}

\subsection{Normal physiology}

The normal motility of gut is maintained by the complex interaction among the central nervous system, enteric nervous system, and hormonal and local factors directly acting on the intestinal smooth muscle (Livingston and Passaro 1990; Mattei and Rombeau 2006). The autonomic nerve system consists of parasympathetic, sympathetic and enteric nervous systems. It has a profound effect on gut motility, ion transportation and is involved in secretory and absorptive processes within GIT. The parasympathetic part of the nervous system is provided by vagus (proximal GIT) and pelvic parasympathetic (distal GIT) nerves. These nerve fibres have a stimulating effect on GIT. The sympathetic innervation is provided by the splanchnic nerve. These post-ganglionic fibres run along the arteries and synapse with the myenteric plexus on the gut wall. The sympathetic nerve fibres have an inhibitory effect on gut which includes inhibition of secretions and motility, and constriction of sphincters and blood vessels. The animal models have shown the sympathetic control is dominant over parasympathetic (Livingston and Passaro 1990).

The enteric nervous system, which is also known as the intrinsic nervous system, consists of myenteric and submucosal plexuses. It makes an important contribution in controlling GI motility and coordinating relaxation and contraction of smooth muscle. The myenteric plexus mainly controls motility of the digestive tract, whilst the submucosal plexus mainly regulates the gastrointestinal blood flow and epithelial function. The enteric nervous system consists of sensory, motor and interneurons. Acetylcholine is the major neurotransmitter which is produced by enteric neurons. Although, the enteric nervous system has the ability to function independently, for normal digestive process it links with the central (extrinsic) nervous system. Due to these cross connections gut provides sensory information to the central nervous system and therefore it may recieve efferent signals from it (Boeckxstaens and de Jonge 2009; Luckey, et al. 2003; Wood 2008). In addition, various hormones and enzymes play an important role in gut function and its motility. The gastrointestinal tract is the largest endocrine organ, called enteric endocrine system, with endocrine glands diffusely scattered throughout the GIT. The hormones secreted by these glands work by a negative feedback mechanism. The enteric endocrine system and nervous systems (enteric and autonomic nervous systems) work in a coordinated way to maintain 
the regular contractions of smooth muscle. It has two specific patterns: slow wave and spike potentials. The spike potentials are true action potentials which trigger the muscle contraction. The motility of stomach and small bowel varies according to fed and fasting states. Further, in fed state the number of contractions, their intensity and duration are based on the food ingested. During fasting state the migrating motor complex (MMC) dictates the pattern of bowel contraction (Luckey, et al. 2003). Due to MMC, the remnants of meal, bacterial and other debris are pushed into the large intestine. Hence they have housekeeping function and prevent bacterial overgrowth in the small intestine. Thus any imbalance in autonomic nerves system, enteric endocrine and the local environment may impair the normal gut motility and digestive process, and may lead to ileus.

\subsection{Pathophysiology}

Ileus is caused by temporary inhibition of extrinsic motility regulation and is more prominent in the colon, while paralytic ileus involves all or part of the GIT and is caused by the inhibition of local and intrinsic contractile systems along with extrinsic inhibition (Bauer and Boeckxstaens 2004). Hence each local factor, autonomic nervous system and hormonal effect has an independent role in the development of POI.

In the past, surgical stress was considered to cause POI by increasing serum catecholamine concentration which would inhibit the gut motility and augment sympathetic activity (Smith, et al. 1977). However, experiments on animal models have shown that although adrenalectomy reduces the level of catecholamine, it does not improve POI. In contrast, splanchnicectomy partially improves ileus (Dubois, et al. 1975). These findings support the hypothesis that the sympathetic nervous system is independent of adrenal activity in causing ileus (Livingston and Passaro 1990). Similarly, vasopressin inhibits intestinal motility (Mitchell and Collin 1985). It is released as a part of stress response following surgical trauma and after the use of opiates (Cochrane, et al. 1981); (Weiskopf, et al. 1987). The exact mechanism by which vasopressin mediates dysmotility is not clear. However, it is suggested that vasopressin is related to reduction in mesenteric blood flow which may subsequently cause impairment of gastrointestinal motility (Livingston and Passaro 1990).

Evidence suggests that three major mechanisms play important role in the development of POI, namely; neurogenic, inflammatory and pharmacological mechanisms. Either of these can cause ileus independently, as does their cumulative effect (Bauer and Boeckxstaens 2004).

\subsubsection{Neurogenic mechanisms}

In 1872, Golz suggested that intestinal contractility increases after division of the neural axis. This was one of the first observations suggesting the presence of inhibitory spinal reflexes (Bauer and Boeckxstaens 2004). Later evidence has shown that splanchnicectomy improves bowel contraction after laparotomy. It was further suggested that splanchnic afferents are largely involved during gastrointestinal surgery, rather than vagal afferents, leading to hypomotility after surgical intervention (Bauer and Boeckxstaens 2004).

Recent investigations found that different neural pathways are activated during abdominal surgery. Their pathways depend upon the part of GIT involved and the intensity of nociceptive stimulus. For instance, skin incision and laparotomy activate an adrenergic 
inhibitory pathway, which is a low threshold spinal reflex and reduces gut motility (Boeckxstaens, et al. 1999). Strong stimuli, such as manipulation of the intestine, may activate an additional high-threshold supraspinal pathway (Barquist, et al. 1996). The corticotrophin-releasing factor (CRF) also plays a central role in this pathway. CRF antagonist has been shown to prevent gastric ileus (Bonaz and Tache 1997). Along with these there are non-adrenergic, vagally mediated, inhibitory pathways triggered by intense stimulation of splanchnic afferent fibres (Boeckxstaens, et al. 1999). Animal models have shown that nitric oxide (NO) is a most potent neurotransmitter in non-adrenergic pathways (Boeckxstaens, et al. 1999; De Winter, et al. 1997). Similarly, vasoactive intestinal peptide (VIP) acts as an inhibitory neurotransmitter during surgical stress (Boeckxstaens, et al. 2000; De Winter, et al. 1998).

Other studies have shown that $\mu$-opioid receptor agonists (e.g morphine) prolong the duration of POI and k-opioid receptor agonists, such as fedotosine, resolve ileus through a peripheral action on non-vagal sensory afferent (Gue, et al. 1989). It is suggested that activation of k-opioid receptors on splanchnic afferent reduces POI by decreasing visceral nociception and inhibitory reflexes. The neural mechanism is primarily activated during the first few hours after surgery and cannot account of POI that lasts for days (Holte and Kehlet 2000; Reissman, et al. 1996).

\subsubsection{Inflammatory mechanisms}

The inflammatory response develops mainly at a local level and is associated directly with the development of POI (Bauer and Boeckxstaens 2004). For this local inflammatory response, muscularis externa provides a unique immunological compartment containing a rich network of macrophages (Faussone-Pellegrini, et al. 1990; Mikkelsen 1995). These macrophages secrete a number of inflammatory mediators such as cytokines, nitric oxide (NO), prostaglandins, and defensins (Cicalese, et al. 1996; Kagan, et al. 1994). Surgical manipulation of bowel activates the macrophages in muscularis externa stimulating release of above inflammaotory mediators (Kalff 1999; Schwarz, et al. 2001).

Intestinal manipulation also provokes the release of tumour necrosis factor- $\alpha$ (TNF- $\alpha$ ), monocyte chemotactic protein-1, interleukin- $1 \mathrm{~b}$ and interleukin- 6 . These pro-inflammatory cytokines up-regulate adhesion molecules, such as intercellular adhesion molecule-1 (ICAM-1). This molecular response expands to cellular level and recruits more leucocytes (monocytes and neutropils) into the muscularis externa that further release NO, cytokines, reactive oxygen intermediates and proteases (Kalff, et al. 1999). The role of NO and prostaglandins in the development of POI has been shown in pharmacological and genetic studies ((Kalff 1999; Schwarz, et al. 2001). There is strong association between neural and inflammatory mechanisms as discussed below.

\subsubsection{Inflammatory-neuronal interaction}

It is well established that intestinal manipulation mediates local inflammation which causes inhibition of smooth muscle function. The manipulation of the small bowel not only causes ileus in the small bowel, it is also linked with delayed gastric emptying (de Jonge, et al. 2003). This raises the question as to whether there is any other mechanism which affects motility distant from the site of inflammation or manipulation? Bauer and Boeckxstaens (2004), suggested that: 
"One possible mechanism could be an interaction between the inflammatory milieu of the postoperative muscularis and primary afferent neuronal activity, triggering the neural inhibitory pathways".

Animal models show that the delay in gastric emptying is induced by intestinal manipulation which occurs through an inhibitory adrenergic pathway. Up-regulation of cyclooxygenase-2 (COX-2) releases prostaglandins that participate in local inflammation and the development of POI (Schwarz, et al. 2001). A number of experimental studies have suggested that prostaglandins act as neuromodulators and their levels increase significantly following surgery. Animal models treated with selective COX-2 inhibitors show a reduction in incidence of ileus. Based on the findings of such studies Bauer and Boeckxstaens (2004) also concluded that:

"the intestinal inflammation in response to bowel manipulation results in primary afferent activation, initiating subsequent inhibitory motor reflexes to the gut, and leading to postoperative intestinal gut dysfunction, with prostaglandins playing a crucial role".

Furthermore, intestinal manipulation activates mast cells which play a vital role in the development of inflammation. During surgery even a gentle manipulation of intestine triggers the release of mast cell mediators. The activated mast cells release potent pro-inflammatory mediators such as TNF- $\alpha$, protease and histamine which contribute to the inflammatory response. In addition, these mediators recruit more leucocytes and up-regulate adhesion molecules, all taking part in inflammatory process (Kubes and Kanwar 1994; Wershil, et al. 1996). The activated mast cells briefly increase intestinal permeability resulting in translocation of bacteria and consequently activation of resident macrophages in the muscularis externa. An inhibitory adrenergic neural pathway is also activated which impairs the neuromuscular function of the distant part of intestine, explaining the generalised features of ileus (Boeckxstaens and de Jonge 2009). Studies have demostrated that mast cell stabilizers improve gastric emptying and reduce inflammatory response (de Jonge, et al. 2003). Hence mast cells and resident macrophages are mainly responsible for activation of the innate immune system, leading to an inflammatory response after intestinal manipulation.

\subsubsection{Pharmacological mechanisms}

There are two types of opiates: endogenous and exogenous. Both play an important role in gastrointestinal dysmotility. Both are simliar and exert their actions through same opoid receptors (Prasad and Matthews 1999). Overall, opiates have an inhibitory effect which leads to impairment in gut motility, its secretions, and the transport of fluid and electrolytes across the gut wall. Understandibly, such actions lead to a delay in gastric emptying and inhibition of intestinal peristalsis and the development of POI (Kurz and Sessler 2003). This phenomenon has been proven in a number of studies on animal and human models which have shown that $\delta$ and $\mu$ opioid agonists diminish peristalsis (Bauer, et al. 1991; Bauer AJ 1991).

There are three different types of opoid receptors: $\delta$ (delta), $\mu$ (mu) and $к$ (kappa). Opiates have a receptor-specific effect on intestine and hence they mainly affect $\mu$ - receptors on GIT, while it has less effect on other organs receptors such as the brain or spinal cord. This is why morphine has more of a constipating effect than an analgesic effect. The requirement of morphine is four times more likely to increase to have an analgesic effect than constipating effect (4:1 ratio) (Holte and Kehlet 2000; Kalff, et al. 1998). Therefore, when opiates are used 
repeatedly, patients develop a tolerance to analgesic effect but not to the side effects on GIT (Holte and Kehlet 2000). A prospective study performed by Cali and colleagues did not show any significant correlation between the length of incision and the dose of morphine taken. However, it suggested that the dose of morphine is directly related to the return of gut function (Cali, et al. 2000). Similarly, opiates bind to leucocytes and affect the immune system which slows down gut motility. They also facilitate NO synthesis which plays a significant part in the pathogenesis of POI. Release of various hormones also plays a role in the pathogenesis of ileus. For example, corticotrophin-releasing factor stimulates inflammatory mediators in the bowel (Tache, et al. 1993).

In summary, there are multiple factors which contribute to the pathogenesis of POI and major mechanisms include neurogenic, inflammatory, pharmacological and hormonal responses (figure 1).

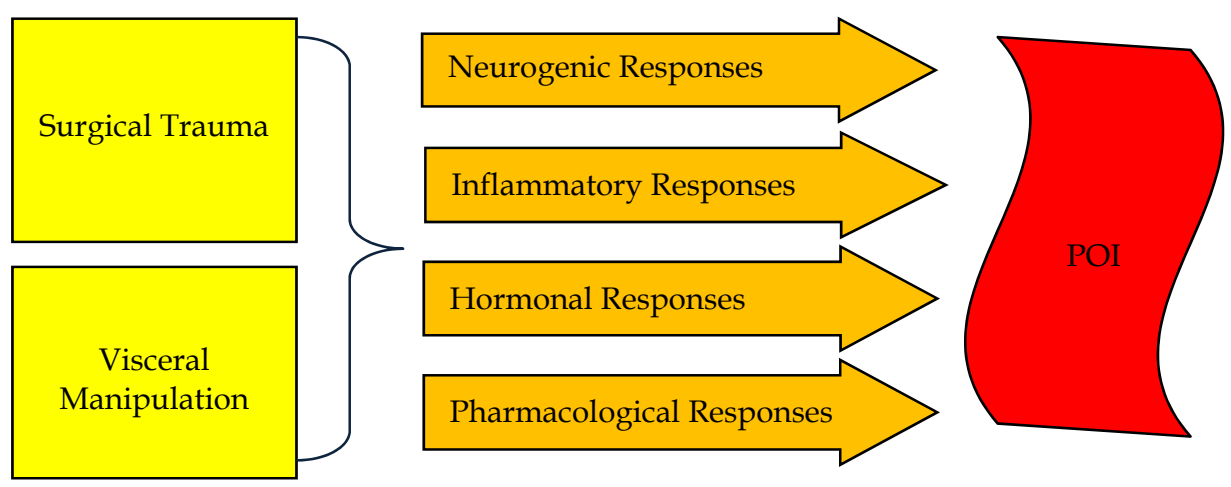

Fig. 1. Pathogenesis of post-operative ileus

\section{Strategies to reduce POI}

Over the past two decades, a number of strategies have been introduced to improve the quality of peroperative care. Most of those were primarily directed at reducing perioperative mortality, morbidity and LOS in hospital. All of those were aimed to prevent ileus by minimising the stress response and maintaining the normal body physiology during and after surgery. These include ERAS programmes, laparoscopic surgical approaches and the use of specific pharmaceutical agents. In this section, different strategies to prevent and manage POI are discussed.

\subsection{Modalities to manage POI}

\subsubsection{Enhanced Recovery After Surgery (ERAS) protocols}

ERAS protocols involve a series of measures taken pre-, per- and post-operatively which collectively ease the stress response to surgical trauma and enhance post-operative recovery. It has been shown that this 'multimodal rehabilitation' programme improves surgical outcome and patient satisfaction after elective surgery (Khoo, et al. 2007; Varadhan, et al. 2010; Wind, et al. 2006b). ERAS programme consists of a number of elements which are summarised in Figure- 2. 


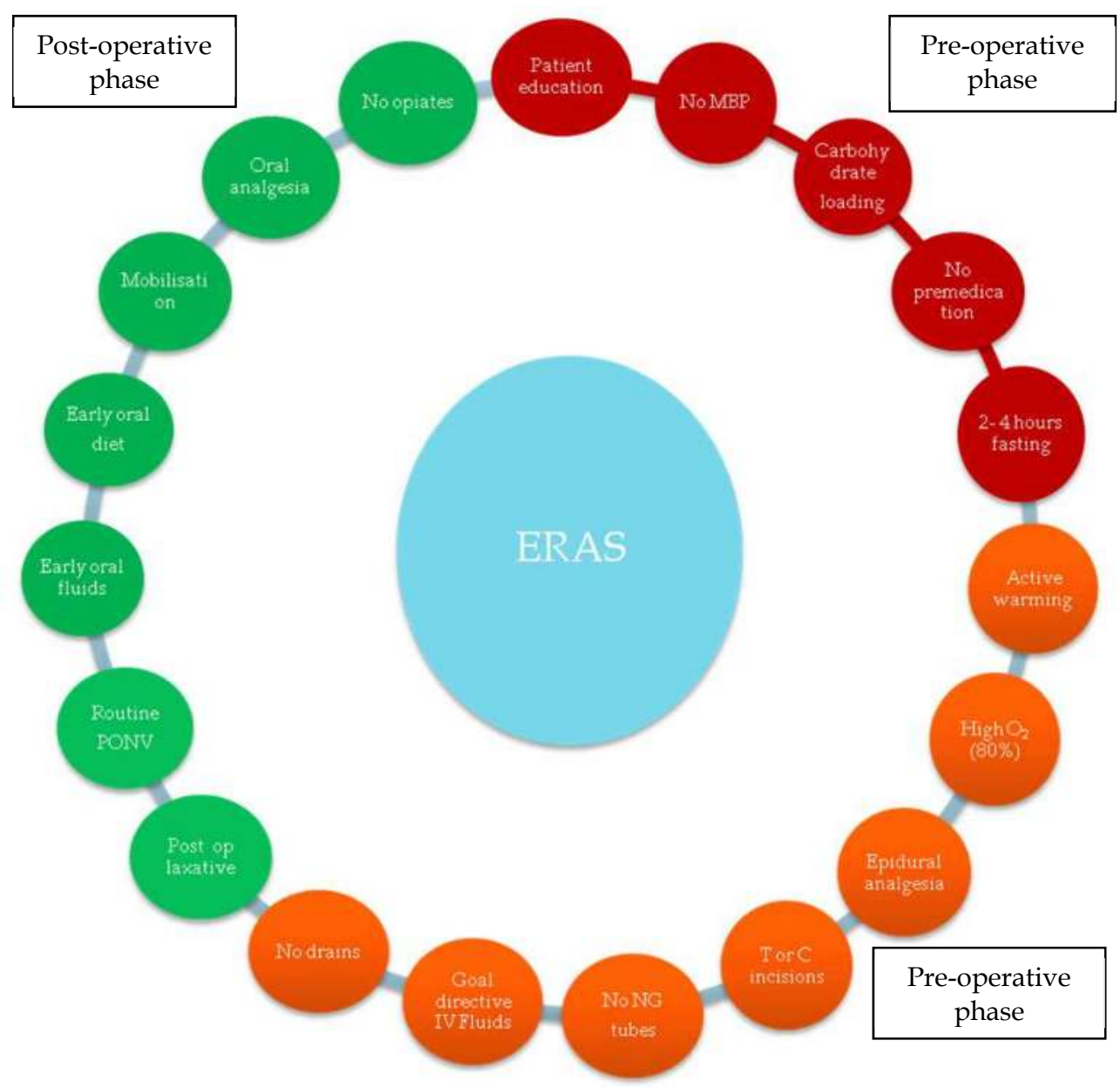

Fig. 2. MBP- Mechanical Bowel Preparation, T and C- Transverse and curve , NG- Nasogastric, PONV- prevention of nausea and vomiting. (Authors previously published this figure as Ahmed J et al, Colorectal Disease, 2011 Oct 11. doi: 10.1111/j.1463- 5 1318.2011.02856.x.)

\subsubsection{Pre-operative components of ERAS protocols}

Preoperative patient counselling and education prior to surgery is important aspect of ERAS protocols. Along with counselling, patients should also see other members of the multidisciplinary team such as dieticians, stoma care nurses and nutrition nurses. Recently, a number of centres have introduced a dedicated ERAS nurse who provides information regarding ERAS pathways and the various stages during and after surgery. An important component of preoperative phase is the ingestion of carbohydrate-rich drink the night before and on the morning of surgery. A short period of fasting and carbohydrate loading both help in maintaining patients' nitrogen balance and reducing post-operative insulin resistance (Soop, et al. 2004; Svanfeldt, et al. 2007). 
Oral mechanical bowel preparation should be avoided in patients undergoing elective bowel surgery. A single phosphate enema can be used on the days of surgery to clear the rectum in patients undergoing left-sided anastomosis. A Cochrane review suggested that low molecular weight heparin (LMWH) and graduated compression thromboembolic deterrent stockings (TEDs) are the most effective anti-thrombotic prophylaxis (WilleJorgensen, et al. 2003). A single dose of broad spectrum antibiotics reduces the risk of wound infection and should be administered prior to skin incision.

\subsubsection{Peri-operative components of ERAS protocols}

High inspired oxygen concentrations $(80 \%)$ should be administered during anaesthesia and in the early post-operative period (for a minimum of six hours). Oxygen molecules are required for the production of free radicals, which play an important role in the defence mechanism against pathogens to reduce surgical site infection, and in the synthesis of collagen for wound healing and angiogenensis (Allen, et al. 1997; Hopf, et al. 1997; Qadan, et al. 2009). In addition, high concentration of oxygen in the tissues during the immediate post-operative period improves perfusion at the anastomotic site and may also be associated with a reduction in post-operative nausea and vomiting (PONV) (Greif, et al. 1999; Orhan-Sungur, et al. 2008).

The core temperature should be measured and monitored using an oesophageal probe. In patients undergoing prolonged surgery (i.e., more than one hour) warmed intravenous fluid should be administered (Lindwall, et al. 1998; Smith, et al. 1998).

Hypothermia (core temperature less than $36^{\circ} \mathrm{C}$ ) causes tissue hypoxia due to peripheral vasoconstriction which increases the risk of coagulopathy, wound infections and cardiac complications (Frank, et al. 1997; Ozaki, et al. 1995; Schmied, et al. 1996). It is also linked to a change in immune response and an increase in catabolic response (Frank, et al. 1995). Prevention of hypothermia in the peri-operative phase has been shown to decrease blood loss, and cardiac and infective complications (Melling, et al. 2001; Wong, et al. 2007).

Goal directed intra-operative intravenous fluid administration has shown positive impact on early return of gut function and reduction in length of stay in hospital. Oesophageal Doppler or LiDCO plus ${ }^{\mathrm{TM}}$ and LiDCO rapid $^{\mathrm{TM}}$ are minimally invasive methods used to measure the cardiac output and stroke volume. These devices enable the anaesthetist to tailor the fluid requirement (goal directed) according to changes in cardiac output peroperatively. Goal directed fluid therapy has been shown to result in early return of gastric motility, reduction in time to defecation and an improved tolerance to oral feed due to reduced gut oedema (Gan, et al. 2002; Lobo, et al. 2002; Nisanevich, et al. 2005) . Splanchnic hypoperfusion, which may remain unnoticed with traditional monitoring, results in delayed return of gut function. It may lead to bacterial translocation across the gut wall and sepsis (Mythen and Webb 1994). Similarly, an excessive amount of intravenous fluid during surgery may cause the development of gut oedema which may also result in the delay in return of gut function (Grocott, et al. 2005).

The concept of minimally invasive surgery is recommended which includes both open (small and appropriate incisions) as well as laparoscopic approach. In open approach, a transverse, limited midline, oblique or paramedian incision should be used whenever possible. Such small transverse or mini-laparotomy incisions may have positive impact on analgesic requirement, pulmonary complications, systemic inflammatory response and length of stay in hospital 
(Nakagoe, et al. 2001; Nakagoe, et al. 2004; Takegami, et al. 2003; Werawatganon and Charuluxanun 2005). In ERAS protocol, routine use of nasogastric tubes and abdominal drains following GI surgery is not recommended. A Cochrane review (33 studies, 5240 patients) has suggested that there was an earlier return of gut function, reduction in pulmonary complications and an insignificant trend towards increased risk of wound infection in patients not having routine nasogastric tube. However, no difference in the rates of anastomotic dehiescence was demonstrated. Its use is also associated with pain and discomfort to patient and impedes post-operative mobility (Nelson, et al. 2007; Nelson, et al. 2005). Similarly, several randomised studies and meta-analyses have suggested that the routine use of drains does not confer any clinical advantage in the early detection of anastomotic leak or intra-abdominal collection (Jesus, et al. 2004; Karliczek, et al. 2006; Urbach, et al. 1999).

It is recommended that all patients undergoing open abdominal surgery should have high thoracic epidural analgesia. Patient should receive epidural analgesia for at least 12 hours to a maximum of 48 hours. Ideally weaning form epidural should start 12 hours after operation.

However, in laparoscopic colorectal surgery the benefits of epidural analgesia are not clear. It may be used depending upon the preference of the operating surgeon and anaesthetist. The epidural anaesthesia blocks sympathetic reflexes and afferent stimuli. This attenuates the post-operative stress and promotes early return of gut function which reduces incidence of PIO (Holte and Kehlet 2002; Marret, et al. 2007). It is important to note that epidural analgesia may cause hypotension due to vasodilatation which may have an adverse affect on the anastomosis. Early recognition of these patients and monitoring in the high dependency unit is therfore critical.

Recently, other modalities such as transversus abdominis plane (TAP) blocks and wound irrigation (PainBuster $\left.{ }^{\circledR}\right)$ with local anaesthetic $\left(0.2 \%\right.$ ropivacaine $\left.{ }^{\circledR}\right)$ have shown a reduction in use of opiates and improvement in patient mobilisation in the early post-operative period (Bamigboye and Hofmeyr 2009; Beaussier, et al. 2007).

\subsubsection{Post-operative components of ERAS protocols}

Opiates have a profound negative effect on the gut motility, consequently delaying return of gut function and prolonging the duration of POI. Regular use of Paracetamol and nonsteroidal anti-inflammatory drugs (NSAIDS) is recommended unless contraindicated. NSAIDS help promote gut recovery by reducing local mucosal inflammation (Cali, et al. 2000). Prophylactic antiemetics should be prescribed to those patients receiving opiates.

It is emphasised that all patients be allowed oral fluid and diet soon after surgery. Intravenous fluid should be stopped as soon as the patient's oral intake is adequate. There is now evidence to support the view that early commencement of enteral feeding is beneficial and is safe to introduce within 24 hours of surgery (Andersen, et al. 2006; Lewis, et al. 2009). Similarly, early feeding facilitates gut motility and may help in the early return of gut function and reduce the ileus.

All patients should have an enforced and structured mobilisation plan during the postoperative period. A designated physiotherapist with a mobilisation plan tailored to the individual patient's need should work closely with the patient in order to achieve best outcome. Early mobilisation is paramount in preventing venous thromboembolism (VTE), respiratory complications and reduction in muscle strength (Schuster and Montie 2002). It is 
noteworthy that early post-operative ambulation plays a little role in preventing or resolving POI. However, it has proven benefits in preventing atelectasis, pneumonia and VTE (Carroll and Alavi 2009) which may cause secondary POI.

A clinical management pathway, based on ERAS protocols, is proposed below which would be useful for colorectal surgeons in day-to-day management of surgical patients (Figure- 3 ).

A typical pathway for management of patients after colorectal surgery - Based on multimodal optimisation

\section{Preoperative Phase}

Patient counselling and education (both written and verbal)

Assessment by Anaesthetist and ERAS Nurse

Optimisation of nutritional status (MUST scoring) and co-morbidities

Stoma education and training (if required)

Carbohydrate loading

Clear fluids till 2 hours prior to surgery

Bowel prep (if required)

Preoperative assessment

VTE prophylaxis

No premedication

Peroperative Phase

Epidural analgesia

Goal directed IV fluids (Use Oesophageal Doppler)

No nasogastric tube or drains

Prophylactic antibiotics

Minimally invasive surgical approach (laparoscopic/ small / transverse incision)

\section{Postoperative Phase}

Set of daily goals for postoperative care

Start oral fluids and diet as soon as patient tolerates

Regular paracetamol and NSAID if not contraindicated

Continue high energy drinks

Avoid regular Morphine and other opiates

Daily review by physio and active and enforced mobilisation

Fig. 3. Clinical management pathway based on ERAS protocols 


\subsection{Laparoscopic approach}

Introduction of laparoscopic approach has significant imapct on surgical practice, for both the surgeons and patients alike. It is known that the laparoscopic techniques cause less surgical stress and trauma, and the patients recover faster (Bauer and Boeckxstaens 2004; Kalff, et al. 1998). Laparoscopic surgery involves less bowel manipulation and inflicts reduced tissue trauma compared with open approach. Similarly, reduced inflammatory and catabolic responses in laparoscopic approach may also result in early recovery after operation. Therefore, the return of gut function is faster compared to open surgery. Effective postoperative analgesia may be obtained with simple analgesics and use of opiates can easily be avoided. Understandably, laparoscopic technique is considered to reduce severity and duration of ileus resulting in shorther length of stay in hospital (Leung, et al. 2004).

Laparoscopic technique has attracted ethusiasm and application by most surgical specialities and has become standard approach for a number of surgical procedures. Its wide spread popularity is based on the short term benefits. Randomised studies have demonstrated that laparoscopic approach reduces duration of POI after abdominal surgery (Basse, et al. 2003; Lacy, et al. 1995; Milsom, et al. 1998). Similarly, laparoscopic colorectal resections have been shown to cause less post-operative pain, and early return of organ function and discharge from hospital when compared with conventional open surgery (Reza, et al. 2006).

Whereas individual trials demostrate short term benefits of laparoscopic approach especially in early postoperative period (Guillou, et al. 2005; Murray, et al. 2006; Veldkamp, et al. 2005), a Cochrane review found no difference in long term outcomes compared with open approach (Kuhry, et al. 2008). However, it is important to consider that most of above evidence is collected from studies lacking ERAS programme of perioperative optimisation. Recently, two trials investigated impact of laparoscopic approach within ERAS application (Basse, et al. 2005; King, et al. 2006). Unfortunately, the results of these two trials are not consistent. Basse et al. failed to show any advantage of laparoscopic approach in colonic resections, while King et al suggested benefits of laparoscopic approach compared with open techniques. However, latter trial was not blinded which might have impacted on the results of the study. Nevertheless, it is established that laparoscopic surgery, in general, is associated with reduced post operative pain and inflammatory response. Consequently, it may play a role in reducing the incidence of POI (Story and Chamberlain 2009). More work is underway in this area and evidence is eagerly awaited.

\subsection{Pharmacological therapies}

There are a number of pharmacological therapies which are part of ERAS protocols. For instance, carbohydrate loading, use of NSAID and prophylactic anti-emetics, epidural and/or regional analgesia are integral part of ERAS pathways. These therapeutic modalities have been shown to have significant impact on early return of gut function (Kehlet and Wilmore 2008). Moreover, it is possible to avoid opiates which have a profound effect on gut motility and cause ileus.

A number of new pharmacological agents are under investigation at different levels. For example, Alvimopan, a selective Mu-opioid receptor antagonist which acts peripherally, has 
been shown to have positive effect on the ileus in phase III trail. It has been shown to have an association with early return of bowel function, reduced incidence of POI, and reduction in length of hospital stay (Ludwig, et al. 2010). Similarly, estimated hospital cost was reduced by $\$ 879$ - $\$ 977$ for patients who received alvimopan compared with placebo. Longterm safety, accurate indications and dosage are important issues which need addressing before its widespread use in future. However, it is unknown that if alvimopan is more cost effective than optimal use of laparoscopic approach and ERAS protocols, and further research is needed (Stewart and Waxman 2010).

Similarly, vagal stimulation by enteral administration of lipid-rich nutrition has shown early recovery of gut peristalsis (Lubbers, et al. 2010). Other agents such as peripheral opioid antagonist methylnaltrexone, motilin analogue atilmotin and Cisapride have been used with promising results (Livingston and Passaro 1990). Adrenergic blockers and parasympathetic agonists (e.g neostigmine) have also been investigated as potential options in treating POI. Unfortunately, these agents have serious side effects and hence not suitable for routine practice. Moreover, drugs such as Erythromycin and Metoclopramide have shown some favourable results in early gastric emptying, however, there is no quality data to support their routine use at a larger scale in daily practice (Sustic, et al. 2005; Yeo, et al. 1993). A recent review suggested that gum chewing in postoperative period is associated with reduction in POI, likely due to vagal stimulation of the gastrointestinal tract (Fitzgerald and Ahmed 2009).

The pharmacological management of POI is still evolving and has an important role in the management of POI. Further evidence is required to establish the summative effect of pharmacological therapies, laparoscopic surgery and ERAS pathways.

\section{Conclusion}

POI is a complex condition of multifactorial aetiology, most commonly seen following abdominal surgery. It causes significant frustration amongst health professionals and patients alike. It is one of the commonest causes of delay in patients' postoperative recovery and carries financial implications due to prolonged length of stay. It is estimated to cost $\$ 1.28$ billion annually in United States (Senagore 2007).

In recent years, efforts have been made to address this issue albeit without any major progress. Nonetheless, our understanding of pathophysiology of ileus is better than before and a number of strategies to overcome the causative factors have been tried (Stewart and Waxman 2010; Story and Chamberlain 2009). Although such strategies help reduce the duration of ileus, they have limited role in preventing its onset (Lubawski and Saclarides 2008). Three major mechanisms - neurogenic, inflammatory and pharmacological - have been implicated in causing ileus (Augestad and Delaney 2010). The level of activation of these factors varies during a surgical procedure. Moreover, understanding of pharmacological mechanisms has opened new windows for research and interventions in this area.

Evidence has accumulated to suggest enhanced recovery after surgery protocol, laparoscopic approach, and pharmacological interventions all reduce the incidence of ileus. Figure 4 illustrates a suggested pathway for prevention and management of prolonged Ileus. 


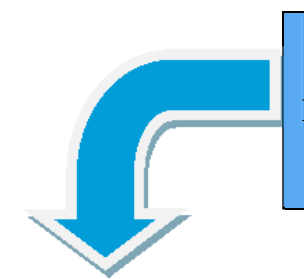

Prevention of POI

Perioperative optimisation

\section{Post Operative Ileus}

Prevention and clinical management pathway

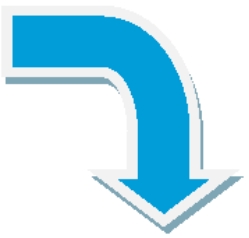

Management of Prolonged POI
Mechanical obstruction must be excluded with contrast studies

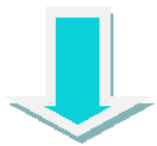

Implementation of ERAS protocols

Nutritional support watchful waiting and supportive treatment

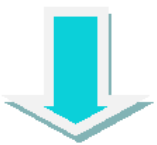

Use of minimally invasive surgical

Nil per mouth

approach

Consider use of pharmacological

Consider nasogastric tube

therapy

Intravenous fluids

Correct electrolytes imbalance

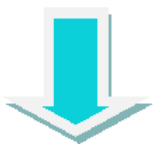

Prevention of complications such as chest infection, heart failure, urinary infection, intrabdominal sepsis
Treat underlying cause i.e. sepsis , reduce opiates use

Patient developing gut failure consider artificial nutrition through central line

Fig. 4. Management pathway for Prolonged POI 
Laparoscopic surgery produces better short term results compared with open approach (Guillou, et al. 2005; Hewett, et al. 2008). Short term morbidity, including incidence of ileus, is significantly low in patients treated with laparoscopic approach. It remains an area of significant reserach interest to investigate whether the combination of laparoscopic surgery and ERAS protocol will offer any further clinical benefit. The ongoing LAFA (Dutch) and EnRoL (UK) trials are desinged to answer above question when completed (Wind, et al. 2006a).

Of above, ERAS pathway was most extensively studied intervention over the last 15 years.The components of ERAS protocol are aimed at optimising patient care, reducing operative stress, and enhancing recovery after surgery by persevering normal physiology and early return of gastrointestinal function. Whereas POI has not been investigated as sole primary endpoint in studies comparing ERAS verusu conventional perioperative care, it has been studied as a part of morbidity rates in both as primary and secondary endpoints. There is now evidence that ERAS protocol of perioperative care is associated with significant reduction in incidence of POI, along with length of hospital stay, compared with conventional perioperative care.

Pharmacological interventions, as part of ERAS or outside ERAS, play key role in reduction of incidence of ileus. Such interventions with promising results include use of preoperative carbohydrate loading, NSAIDs, prophylactic anti-emetics, and epidural and/or regional analgesia. Avoidance of opiates and use of mu-opoid receptor antagonists are associated with early return of gut function.

The impact of ileus as an important health issue cannot be over emphasized:

"In patients who underwent colectomy surgery, postoperative ileus was associated with a $29 \%$ increase in hospital LOS and a 15\% increase in hospitalization costs. Prevention of postoperative ileus could potentially yield benefits in reduction in hospital LOS and associated health care costs." (Lyer, et al. 2009)

POI is a challenging yet preventable event in surgical patients. It has a multifactorial pathophysiology that requires a multimodal approach in its management. Whereas there is no definitive treatment of ileus, strategies to prevent and manage ileus should be followed as discussed above. Essentially, prevention and management of POI centres on minimising perioperative stress and preserving normal physiological functions.

\section{References}

2004 A comparison of laparoscopically assisted and open colectomy for colon cancer. N Engl J Med 350(20):2050-9.

Ahmed, J., et al. 2010 Compliance with enhanced recovery programmes in elective colorectal surgery. Br J Surg 97(5):754-8.

Allen, D. B., et al. 1997 Wound hypoxia and acidosis limit neutrophil bacterial killing mechanisms. Arch Surg 132(9):991-6.

Andersen, H. K., S. J. Lewis, and S. Thomas 2006 Early enteral nutrition within $24 \mathrm{~h}$ of colorectal surgery versus later commencement of feeding for postoperative complications. Cochrane Database Syst Rev (4):CD004080.

Asgeirsson, T., et al. 2010 Postoperative ileus: it costs more than you expect. J Am Coll Surg 210(2):228-31. 
Augestad, K. M., and C. P. Delaney 2010 Postoperative ileus: impact of pharmacological treatment, laparoscopic surgery and enhanced recovery pathways. World J Gastroenterol 16(17):2067-74.

Bamigboye, A. A., and G. J. Hofmeyr 2009 Local anaesthetic wound infiltration and abdominal nerves block during caesarean section for postoperative pain relief. Cochrane Database Syst Rev (3):CD006954.

Barquist, E., et al. 1996 Neuronal pathways involved in abdominal surgery-induced gastric ileus in rats. Am J Physiol 270(4 Pt 2):R888-94.

Basse, L., et al. 2005 Functional recovery after open versus laparoscopic colonic resection: a randomized, blinded study. Ann Surg 241(3):416-23.

- 2003 Gastrointestinal transit after laparoscopic versus open colonic resection. Surg Endosc 17(12):1919-22.

Bauer, A. J., and G. E. Boeckxstaens 2004 Mechanisms of postoperative ileus. Neurogastroenterol Motil 16 Suppl 2:54-60.

Bauer, A. J., M. G. Sarr, and J. H. Szurszewski 1991 Opioids inhibit neuromuscular transmission in circular muscle of human and baboon jejunum. Gastroenterology 101(4):970-6.

Bauer AJ, Szurszewski JH. 1991 Effect of opioid peptides on circular muscle of canine duodenum j Physiol 434:409- 422.

Beaussier, M., et al. 2007 Continuous preperitoneal infusion of ropivacaine provides effective analgesia and accelerates recovery after colorectal surgery: a randomized, double-blind, placebo-controlled study. Anesthesiology 107(3):461-8.

Boeckxstaens, G. E., and W. J. de Jonge 2009 Neuroimmune mechanisms in postoperative ileus. Gut 58(9):1300-11.

Boeckxstaens, G. E., et al. 1999 Activation of an adrenergic and vagally-mediated NANC pathway in surgery-induced fundic relaxation in the rat. Neurogastroenterol Motil 11(6):467-74.

- 2000 Evidence for VIP(1)/PACAP receptors in the afferent pathway mediating surgeryinduced fundic relaxation in the rat. Br J Pharmacol 131(4):705-10.

Bonaz, B., and Y. Tache 1997 Corticotropin-releasing factor and systemic capsaicin-sensitive afferents are involved in abdominal surgery-induced Fos expression in the paraventricular nucleus of the hypothalamus. Brain Res 748(1-2):12-20.

Cali, R. L., et al. 2000 Effect of Morphine and incision length on bowel function after colectomy. Dis Colon Rectum 43(2):163-8.

Carroll, J., and K. Alavi 2009 Pathogenesis and management of postoperative ileus. Clin Colon Rectal Surg 22(1):47-50.

Cicalese, L., et al. 1996 Pyruvate prevents mucosal reperfusion injury, oxygen free-radical production, and neutrophil infiltration after rat small bowel preservation and transplantation. Transplant Proc 28(5):2611.

Cochrane, J. P., et al. 1981 Arginine vasopressin release following surgical operations. Br J Surg 68(3):209-13.

de Jonge, W. J., et al. 2003 Postoperative ileus is maintained by intestinal immune infiltrates that activate inhibitory neural pathways in mice. Gastroenterology 125(4):1137-47.

De Winter, B. Y., et al. 1997 Effect of adrenergic and nitrergic blockade on experimental ileus in rats. Br J Pharmacol 120(3):464-8.

- 1998 Role of VIP1/PACAP receptors in postoperative ileus in rats. Br J Pharmacol 124(6):1181-6. 
Delaney C, Kehlet H, Senagore A, et al 2006 Postoperative ileus: profiles, risk factors, and definitions - a framework for optimizing surgical outcomes in patients undergoing major abdominal colorectal surgery. Clinical consensus update in general surgery.:26.

Dubois, A., D. P. Henry, and I. J. Kopin 1975 Plasma catecholamines and postoperative gastric emptying and small intestinal propulsion in the rat. Gastroenterology 68(3):466-9.

Faussone-Pellegrini, M. S., D. Pantalone, and C. Cortesini 1990 Smooth muscle cells, interstitial cells of Cajal and myenteric plexus interrelationships in the human colon. Acta Anat (Basel) 139(1):31-44.

Finney, J. M. 1906 IV. Postoperative Ileus. Ann Surg 43(6):870-904.

Fitzgerald, J. E., and I. Ahmed 2009 Systematic review and meta-analysis of chewing-gum therapy in the reduction of postoperative paralytic ileus following gastrointestinal surgery. World J Surg 33(12):2557-66.

Frank, S. M., et al. 1997 Perioperative maintenance of normothermia reduces the incidence of morbid cardiac events. A randomized clinical trial. JAMA 277(14):1127-34.

- 1995 The catecholamine, cortisol, and hemodynamic responses to mild perioperative hypothermia. A randomized clinical trial. Anesthesiology 82(1):83-93.

Gan, T. J., et al. 2002 Goal-directed intraoperative fluid administration reduces length of hospital stay after major surgery. Anesthesiology 97(4):820-6.

Greif, R., et al. 1999 Supplemental oxygen reduces the incidence of postoperative nausea and vomiting. Anesthesiology 91(5):1246-52.

Grocott, M. P., M. G. Mythen, and T. J. Gan 2005 Perioperative fluid management and clinical outcomes in adults. Anesth Analg 100(4):1093-106.

Gue, M., et al. 1989 Stimulation of kappa opiate receptors in intestinal wall affects stressinduced increase of plasma cortisol in dogs. Brain Res 502(1):143-8.

Guillou, P. J., et al. 2005 Short-term endpoints of conventional versus laparoscopic-assisted surgery in patients with colorectal cancer (MRC CLASICC trial): multicentre, randomised controlled trial. Lancet 365(9472):1718-26.

Hewett, P. J., et al. 2008 Short-term outcomes of the Australasian randomized clinical study comparing laparoscopic and conventional open surgical treatments for colon cancer: the ALCCaS trial. Ann Surg 248(5):728-38.

Holte, K., and H. Kehlet 2000 Postoperative ileus: a preventable event. Br J Surg 87(11):148093.

- 2002 Epidural anaesthesia and analgesia - effects on surgical stress responses and implications for postoperative nutrition. Clin Nutr 21(3):199-206.

Hopf, H. W., et al. 1997 Wound tissue oxygen tension predicts the risk of wound infection in surgical patients. Arch Surg 132(9):997-1004; discussion 1005.

Iyer, S., W. B. Saunders, and S. Stemkowski 2009. Economic burden of postoperative ileus associated with colectomy in the United States. J Manag Care Pharm 15(6):485-94.

Jesus, E. C., et al. 2004 Prophylactic anastomotic drainage for colorectal surgery. Cochrane Database Syst Rev (4):CD002100.

Kagan, B. L., T. Ganz, and R. I. Lehrer 1994 Defensins: a family of antimicrobial and cytotoxic peptides. Toxicology 87(1-3):131-49.

Kalff, J. C., et al. 1999 Surgically induced leukocytic infiltrates within the rat intestinal muscularis mediate postoperative ileus. Gastroenterology 117(2):378-87.

- 1998 Surgical manipulation of the gut elicits an intestinal muscularis inflammatory response resulting in postsurgical ileus. Ann Surg 228(5):652-63. 
Kalff, J. C., Schraut WH, Billiar TR, Simmons RL, Baur AJ, 1999 Roles of inducible nitric oxide synthase in postoperative intestinal smooth muscle dysfunction in rodents. . Gastroenterology 188:316-27.

Karliczek, A., et al. 2006 Drainage or nondrainage in elective colorectal anastomosis: a systematic review and meta-analysis. Colorectal Dis 8(4):259-65.

Kehlet, H., and D. W. Wilmore 2008 Evidence-based surgical care and the evolution of fasttrack surgery. Ann Surg 248(2):189-98.

Khoo, C. K., et al. 2007 A prospective randomized controlled trial of multimodal perioperative management protocol in patients undergoing elective colorectal resection for cancer. Ann Surg 245(6):867-72.

King, P. M., et al. 2006 Randomized clinical trial comparing laparoscopic and open surgery for colorectal cancer within an enhanced recovery programme. Br J Surg 93(3):300-8.

Kubes, P., and S. Kanwar 1994 Histamine induces leukocyte rolling in post-capillary venules. A P-selectin-mediated event. J Immunol 152(7):3570-7.

Kuhry, E., et al. 2008 Long-term results of laparoscopic colorectal cancer resection. Cochrane Database Syst Rev (2):CD003432.

Kurz, A., and D. I. Sessler 2003 Opioid-induced bowel dysfunction: pathophysiology and potential new therapies. Drugs 63(7):649-71.

Lacy, A. M., et al. 1995 Short-term outcome analysis of a randomized study comparing laparoscopic vs open colectomy for colon cancer. Surg Endosc 9(10):1101-5.

Leung, K. L., et al. 2004 Laparoscopic resection of rectosigmoid carcinoma: prospective randomised trial. Lancet 363(9416):1187-92.

Lewis, S. J., H. K. Andersen, and S. Thomas 2009 Early enteral nutrition within $24 \mathrm{~h}$ of intestinal surgery versus later commencement of feeding: a systematic review and meta-analysis. J Gastrointest Surg 13(3):569-75.

Lindwall, R., et al. 1998 Forced air warming and intraoperative hypothermia. Eur J Surg 164(1):13-6.

Livingston, E. H., and E. P. Passaro, Jr. 1990 Postoperative ileus. Dig Dis Sci 35(1):121-32.

Lobo, D. N., et al. 2002 Effect of salt and water balance on recovery of gastrointestinal function after elective colonic resection: a randomised controlled trial. Lancet 359(9320):1812-8.

Lubawski, J., and T. Saclarides 2008 Postoperative ileus: strategies for reduction. Ther Clin Risk Manag 4(5):913-7.

Lubbers, T., W. Buurman, and M. Luyer 2010 Controlling postoperative ileus by vagal activation. World J Gastroenterol 16(14):1683-7.

Luckey, A., E. Livingston, and Y. Tache 2003 Mechanisms and treatment of postoperative ileus. Arch Surg 138(2):206-14.

Ludwig, K., et al. 2010 Alvimopan for the management of postoperative ileus after bowel resection: characterization of clinical benefit by pooled responder analysis. World J Surg 34(9):2185-90.

Marret, E., C. Remy, and F. Bonnet 2007 Meta-analysis of epidural analgesia versus parenteral opioid analgesia after colorectal surgery. Br J Surg 94(6):665-73.

Mattei, P., and J. L. Rombeau 2006 Review of the pathophysiology and management of postoperative ileus. World J Surg 30(8):1382-91.

Melling, A. C., et al. 2001 Effects of preoperative warming on the incidence of wound infection after clean surgery: a randomised controlled trial. Lancet 358(9285):876-80.

Miedema, B. W., and J. O. Johnson 2003 Methods for decreasing postoperative gut dysmotility. Lancet Oncol 4(6):365-72. 
Mikkelsen, H. B. 1995 Macrophages in the external muscle layers of mammalian intestines. Histol Histopathol 10(3):719-36.

Milsom, J. W., et al. 1998 A prospective, randomized trial comparing laparoscopic versus conventional techniques in colorectal cancer surgery: a preliminary report. J Am Coll Surg 187(1):46-54; discussion 54-5.

Mitchell, A., and J. Collin 1985 Vasopressin effects on the small intestine: a possible factor in paralytic ileus? Br J Surg 72(6):462-5.

Murray, A., et al. 2006 Clinical effectiveness and cost-effectiveness of laparoscopic surgery for colorectal cancer: systematic reviews and economic evaluation. Health Technol Assess 10(45):1-141, iii-iv.

Mythen, M. G., and A. R. Webb 1994 The role of gut mucosal hypoperfusion in the pathogenesis of post-operative organ dysfunction. Intensive Care Med 20(3):203-9.

Nakagoe, T., et al. 2001 Use of minilaparotomy in the treatment of colonic cancer. Br J Surg 88(6):831-6.

- 2004 Minilaparotomy approach for the resection of laterally spreading tumors of the colon. Surg Today 34(9):737-41.

Nelson, R., S. Edwards, and B. Tse 2007 Prophylactic nasogastric decompression after abdominal surgery. Cochrane Database Syst Rev (3):CD004929.

Nelson, R., B. Tse, and S. Edwards 2005 Systematic review of prophylactic nasogastric decompression after abdominal operations. Br J Surg 92(6):673-80.

Nisanevich, V., et al. 2005 Effect of intraoperative fluid management on outcome after intraabdominal surgery. Anesthesiology 103(1):25-32.

Orhan-Sungur, M., et al. 2008 Does supplemental oxygen reduce postoperative nausea and vomiting? A meta-analysis of randomized controlled trials. Anesth Analg 106(6):1733-8.

Ozaki, M., et al. 1995 Nitrous oxide decreases the threshold for vasoconstriction less than sevoflurane or isoflurane. Anesth Analg 80(6):1212-6.

Parvizi, J., et al. 2008 Postoperative ileus after total joint arthroplasty. J Arthroplasty 23(3):360-5.

Prasad, M., and J. B. Matthews 1999 Deflating postoperative ileus. Gastroenterology 117(2):489-92.

Qadan, M., et al. 2009 Perioperative supplemental oxygen therapy and surgical site infection: a meta-analysis of randomized controlled trials. Arch Surg 144(4):359-66; discussion 366-7.

Reissman, P., F. Agachan, and S. D. Wexner 1996 Outcome of laparoscopic colorectal surgery in older patients. Am Surg 62(12):1060-3.

Reza, M. M., et al. 2006 Systematic review of laparoscopic versus open surgery for colorectal cancer. Br J Surg 93(8):921-8.

Schmied, H., et al. 1996 Mild hypothermia increases blood loss and transfusion requirements during total hip arthroplasty. Lancet 347(8997):289-92.

Schuster, T. G., and J. E. Montie 2002 Postoperative ileus after abdominal surgery. Urology 59(4):465-71.

Schwarz, N. T., et al. 2001 Prostanoid production via COX-2 as a causative mechanism of rodent postoperative ileus. Gastroenterology 121(6):1354-71.

Senagore, A. J. 2007 Pathogenesis and clinical and economic consequences of postoperative ileus. Am J Health Syst Pharm 64(20 Suppl 13):S3-7.

Smith, C. E., et al. 1998 Warming intravenous fluids reduces perioperative hypothermia in women undergoing ambulatory gynecological surgery. Anesth Analg 87(1):37-41. 
Smith, J., K. A. Kelly, and R. M. Weinshilboum 1977 Pathophysiology of postoperative ileus. Arch Surg 112(2):203-9.

Soop, M., et al. 2004 Preoperative oral carbohydrate treatment attenuates endogenous glucose release 3 days after surgery. Clin Nutr 23(4):733-41.

Stewart, D., and K. Waxman

2010 Management of postoperative ileus. Dis Mon 56(4):204-14.

Story, S. K., and R. S. Chamberlain. 2009 A comprehensive review of evidence-based strategies to prevent and treat postoperative ileus. Dig Surg 26(4):265-75.

Sustic, A., et al.2005 Metoclopramide improves gastric but not gallbladder emptying in cardiac surgery patients with early intragastric enteral feeding: randomized controlled trial. Croat Med J 46(2):239-44.

Svanfeldt, M., et al. 2007 Randomized clinical trial of the effect of preoperative oral carbohydrate treatment on postoperative whole-body protein and glucose kinetics. Br J Surg 94(11):1342-50.

Tache, Y., et al. 1993 Role of CRF in stress-related alterations of gastric and colonic motor function. Ann N Y Acad Sci 697:233-43.

Takegami, K., et al. 2003 Minilaparotomy approach to colon cancer. Surg Today 33(6):414-20.

Urbach, D. R., E. D. Kennedy, and M. M. Cohen 1999 Colon and rectal anastomoses do not require routine drainage: a systematic review and meta-analysis. Ann Surg 229(2):174-80.

Varadhan, K. K., et al. 2010 The enhanced recovery after surgery (ERAS) pathway for patients undergoing major elective open colorectal surgery: a meta-analysis of randomized controlled trials. Clin Nutr 29(4):434-40.

Veldkamp, R., et al. 2005 Laparoscopic surgery versus open surgery for colon cancer: shortterm outcomes of a randomised trial. Lancet Oncol 6(7):477-84.

Weiskopf, R. B., et al. 1987 Effects of fentanyl on vasopressin secretion in human subjects. J Pharmacol Exp Ther 242(3):970-3.

Werawatganon, T., and S. Charuluxanun 2005 Patient controlled intravenous opioid analgesia versus continuous epidural analgesia for pain after intra-abdominal surgery. Cochrane Database Syst Rev (1):CD004088.

Wershil, B. K., et al. 1996 Mast cell-dependent neutrophil and mononuclear cell recruitment in immunoglobulin E-induced gastric reactions in mice. Gastroenterology 110(5):1482-90.

Wille-Jorgensen, P., et al. 2003 Heparins and mechanical methods for thromboprophylaxis in colorectal surgery. Cochrane Database Syst Rev (4):CD001217.

Wind, J., et al. 2006a Perioperative strategy in colonic surgery; LAparoscopy and/or FAst track multimodal management versus standard care (LAFA trial). BMC Surg 6:16.

- 2006b Systematic review of enhanced recovery programmes in colonic surgery. Br J Surg 93(7):800-9.

Wong, P. F., et al. 2007 Randomized clinical trial of perioperative systemic warming in major elective abdominal surgery. Br J Surg 94(4):421-6.

Wood, J. D. 2008 Enteric nervous system: reflexes, pattern generators and motility. Curr Opin Gastroenterol 24(2):149-58.

Yeo, C. J., et al. 1993 Erythromycin accelerates gastric emptying after pancreaticoduodenectomy. A prospective, randomized, placebo-controlled trial. Ann Surg 218(3): 229-37; discussion 237-8. 


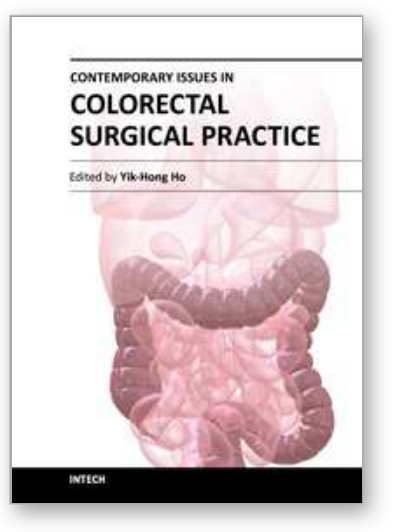

\author{
Contemporary Issues in Colorectal Surgical Practice \\ Edited by Dr. Yik- Hong Ho
}

ISBN 978-953-51-0257-1

Hard cover, 126 pages

Publisher InTech

Published online 16, March, 2012

Published in print edition March, 2012

In recent years, significant progress in colorectal surgery has been made which includes laparoscopic techniques, pre-operative management, emergency colorectal surgery, fast track multimodal recovery, management of complex wound problems and colorectal cancer follow-up. "Contemporary Issues in Colorectal Surgical Practice" aims to bridge the gap between the journal article and the traditional textbook in these areas.

\title{
How to reference
}

In order to correctly reference this scholarly work, feel free to copy and paste the following:

J. Ahmed, S. Mehmood and J. MacFie (2012). Postoperative lleus in Elective Colorectal Surgery: Management Strategies, Contemporary Issues in Colorectal Surgical Practice, Dr. Yik- Hong Ho (Ed.), ISBN: 978-953-510257-1, InTech, Available from: http://www.intechopen.com/books/contemporary-issues-in-colorectal-surgicalpractice/post-operative-ileus-in-elective-colorectal-surgery-management-strategies-

\section{INTECH}

open science | open minds

\section{InTech Europe}

University Campus STeP Ri

Slavka Krautzeka 83/A

51000 Rijeka, Croatia

Phone: +385 (51) 770447

Fax: +385 (51) 686166

www.intechopen.com

\section{InTech China}

Unit 405, Office Block, Hotel Equatorial Shanghai

No.65, Yan An Road (West), Shanghai, 200040, China 中国上海市延安西路65号上海国际贵都大饭店办公楼 405 单元

Phone: +86-21-62489820

Fax: $+86-21-62489821$ 
(C) 2012 The Author(s). Licensee IntechOpen. This is an open access article distributed under the terms of the Creative Commons Attribution 3.0 License, which permits unrestricted use, distribution, and reproduction in any medium, provided the original work is properly cited. 